\title{
Thermally induced spin-transfer torques in superconductor/ferromagnet bilayers
}

\author{
I. V. Bobkova, ${ }^{1,2,3}$ A. M. Bobkov, ${ }^{1}$ and Wolfgang Belzig ${ }^{4}$ \\ ${ }^{1}$ Institute of Solid State Physics, Chernogolovka, Moscow reg., 142432 Russia \\ ${ }^{2}$ Moscow Institute of Physics and Technology, Dolgoprudny, 141700 Russia \\ ${ }^{3}$ National Research University Higher School of Economics, Moscow, 101000 Russia \\ ${ }^{4}$ Fachbereich Physik, Universität Konstanz, D-78457 Konstanz, Germany
}

(Dated: February 8, 2022)

\begin{abstract}
Thermally induced magnetization dynamics is currently a flourishing field of research due to its potential application in information technology. We study the paradigmatic system of a magnetic domain wall in a thermal gradient which is interacting with an adjacent superconductor. The spintransfer torques arising in this system due to the combined action of the giant thermoelectric effect and the creation of equal-spin pairs in the superconductor are large enough to give rise to high domain wall velocities $10^{3}$ times larger than previously predicted.
\end{abstract}

In recent years a new field of research has emerged by coupling spin and heat degrees of freedom - called spin caloritronics [1]. In particular, the spin Seebeck effect, that is the generation of a spin imbalance by a temperature gradient, has been discussed. Further, a thermally induced spin-transfer torque (STT), based on the spindependent Seebeck effect was predicted and its influence on the domain wall (DW) motion has been discussed 2 9. There is also experimental evidence of the thermallyinduced STT in ferromagnetic systems via observations of the magnetization switching and domain wall motion [10 15]. The main mechanisms of the thermal STT are spin transfer via magnons and via thermally-induced electron spin flow.

In this Letter, we propose a paradigm of converting thermal gradients to magnetization dynamics in a very efficient and energy saving way. The key idea is to exploit a superconductor/ferromagnet hybrids as shown in Fig. 1. where the STT is due to the combined action of the giant thermoelectric effect and creation of equal-spin pairs in the superconductor. Our estimates suggest that domain wall velocities of the order of at least a hundred $\mathrm{m} / \mathrm{s}$ can be achieved by extremely small temperature differences smaller than the critical temperature of conventional low-temperature superconductors like $\mathrm{Al}$ and $\mathrm{Nb}$. The efficiency of the thermal STT can be quantified by the ratio of the domain wall velocity $v_{D W}$ to the temperature gradient $\nabla T$. In principle, our estimates give $v_{D W} / \nabla T \gtrsim 10-10^{2} \mathrm{~mm}^{2} /$ Ks for the $\mathrm{S} / \mathrm{F}$ system, which is about three order of magnitude larger than the values $\sim 10^{-2}-10^{-1} \mathrm{~mm}^{2} / \mathrm{Ks}$ reported for thermally induced domain wall motion in ferromagnetic materials [10, 12.

In the framework of the discussed mechanism the thermal STT is provided by the electron spin polarization created in the superconducting part of the structure and subsequent coupling of this polarization to the ferromagnet magnetization via the exchange mechanism. In principle, the STT is universal and can be relevant for ferromagnetic metals as well as for magnetic insulators. The key ingredient for efficient realization of the thermal STT is the Zeeman splitting of the DOS in the superconductor

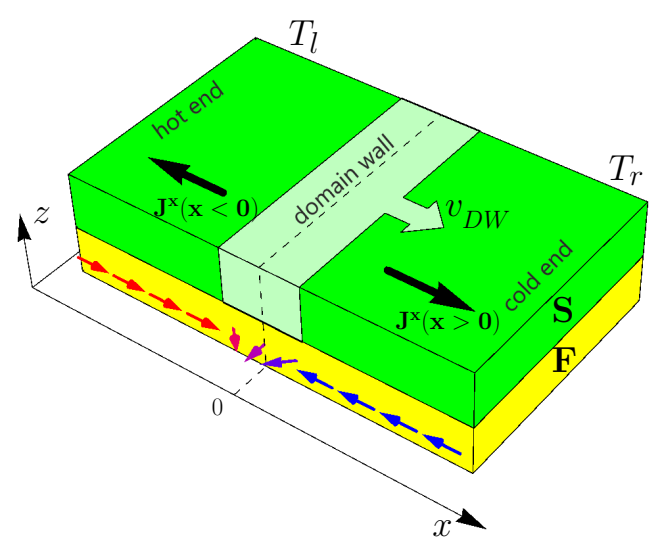

FIG. 1. Sketch of the bilayer S/F system. The magnetization of the ferromagnet $\mathrm{F}$ has a form of a head-to-head domain wall (DW) and is indicated by arrows. The picture on the top surface illustrates the process of thermally induced spin pumping into the DW region. Thermally induced quasiparticles both electron- and hole-like move from the hot to the cold end. In the bulk of both domains the magnetic moments of the quasiparticles are polarized along the corresponding magnetization. Therefore, the spin current (opposite to the magnetization current) in the bulk of both domains is directed away from the DW. In the left (hotter) domain the direction of the majority spin flow is opposite to the spin current direction, while in the right (colder) domain they coincide. Therefore, the spin current flowing in both domains, pumps majority spins of the hotter domain into the DW region. This leads to the expansion of the hotter domain and, consequently, the DW moves from the hot to the cold end.

by proximity to the adjacent ferromagnet. The role of the Zeeman splitting is twofold. First, the presence of superconductivity in the system provides a unique mechanism for an anti-damping spin-transfer torque, which is not connected to spin-flip scattering of quasiparticles: a superconducting quasiparticle spin cannot align itself to the inhomogeneous magnetization at the length scales shorter than the superconducting coherence length $\xi_{S}$ because the quasiparticle strongly interacts with the condensate of equal-spin pairs, where the characteristic 
length scale is $\xi_{S}$. Therefore, if the DW width $l_{D W}$ is less than $\xi_{S}$, the quasiparticle spins are inevitably misaligned to the DW magnetization giving rise to the non-adiabatic torque.

The second contribution to the STT is the thermallyinduced quasiparticle spin flow in the superconducting part (spin-dependent Seebeck effect). This quasiparticle spin flow is known to result in the thermal STT in nonsuperconducting systems $2[9]$. At the same time a Zeeman-split superconductor is a unique example of a physical system, in which a very large spin Seebeck effect can be realized at low temperatures. The spin Seebeck effect can be quantified in terms of the spin thermopower $\nabla \mu_{s} / 2 e \nabla T$ generated by the temperature gradient $\nabla T$ in an open circuit, where $\mu_{s}=\mu_{\uparrow}-\mu_{\downarrow}$ is the spin imbalance of the spin-dependent chemical potentials. In order to have a nonzero spin Seebeck effect an electronhole asymmetry at the Fermi level is required 1, 1620]. Typically the corresponding electron-hole asymmetry in metallic ferromagnets is rather small resulting in the predicted spin thermopower $\mu_{s} / 2 e \nabla T \sim 10^{-3} \mathrm{mV} / \mathrm{K}$ for intermetallic interfaces at room temperature [4, 21, while a much smaller spin thermopower $\sim 10^{-6} \mathrm{mV} / \mathrm{K}$ was measured for a ferromagnetic film 20. In Zeemansplit superconductors a very large thermopower and spin thermopower was predicted [22 33. The experimental observation of the large thermopower has been reported 34 36. Upon application of strong in-plane magnetic fields $B \sim 1 \mathrm{~T}$ or by proximity to a magnetic insulator, Seebeck coefficients of the order of $0.3 \mathrm{mV} / \mathrm{K}$ were measured, which is comparable to the thermopower measured in magnetic semiconductors at much higher temperatures 37 .

Model and method. The model system that we consider is shown in Fig. 1. It consists of a spin-textured ferromagnet with a spatially dependent magnetization $\boldsymbol{M}(\boldsymbol{r})$ in contact to a spin-singlet superconductor. The superconductor is assumed to be in the ballistic limit. The ferromagnet can be a metal or an insulator. If the thickness of the $\mathrm{S}$ film $d_{S}$ is smaller than the superconducting coherence length $\xi_{S}$, the magnetic proximity effect, that is the influence of the adjacent ferromagnet on the $\mathrm{S}$ film can be described by adding the effective exchange field 38 43] $\boldsymbol{h}(\boldsymbol{r}) \sim-\boldsymbol{M}(\boldsymbol{r})$ to the quasiclassical Eilenberger equation, which we use below to treat the superconductor. While in general the magnetic proximity effect is not reduced to the effective exchange only [44-46], in the framework of the present study we neglect other terms which can be viewed as additional magnetic impurities in the superconductor and focus on the effect of the spin texture. The bilayer film is assumed to be connected to equilibrium reservoirs having different temperatures $T_{l, r}$. We neglect all inelastic relaxation processes in the film assuming that its length is shorter than the corresponding relaxation length.

The torque can be calculated starting from the effective exchange interaction between the spin densities on the two sides of the $\mathrm{S} / \mathrm{F}$ interface:

$$
H_{\text {int }}=-\int d^{2} \boldsymbol{r} J_{e x} \boldsymbol{S} \boldsymbol{s},
$$

where $s$ is the electronic spin density operator in the $\mathrm{S}$ film, $\boldsymbol{S}$ is the localized spin operator in the F film, $J_{e x}$ is the exchange constant and the integration is performed over the 2D interface. It has been shown [46] that this exchange interaction Hamiltonian results in the appearance of the exchange field $h=J_{e x} M /\left(2 \gamma d_{s}\right)$ in the S film. Here $M$ is the saturation magnetization of the ferromagnet and $\gamma$ is the gyromagnetic ratio.

The spin density $s$ obeys the following equation:

$$
\partial_{t} \boldsymbol{s}=-\partial_{j} \boldsymbol{J}_{j}-2 \boldsymbol{h} \times \boldsymbol{s},
$$

where we have introduced the vector $\boldsymbol{J}_{j}=\left(J_{j}^{x}, J_{j}^{y}, J_{j}^{z}\right)$ corresponding to the spin current flowing along the $j$-axis in real space.

The additional contribution to the Landau-LifshitzGilbert equation from the exchange interaction Eq. (1) has the form of a torque acting on the magnetization:

$$
\frac{\partial \boldsymbol{M}}{\partial t}=-\gamma \boldsymbol{M} \times \boldsymbol{H}_{\mathrm{eff}}+\frac{\alpha}{M} \boldsymbol{M} \times \frac{\partial \boldsymbol{M}}{\partial t}+\frac{J_{e x}}{d_{F}} \boldsymbol{M} \times \boldsymbol{s},
$$

where $\alpha$ is the Gilbert damping constant and the last term represents the torque. $\boldsymbol{H}_{\text {eff }}$ is the local effective field

$$
\boldsymbol{H}_{\mathrm{eff}}=\frac{H_{K} M_{x}}{M} \boldsymbol{e}_{x}+\frac{2 A}{M^{2}} \nabla^{2} \boldsymbol{M}-K_{\perp} M_{z} \boldsymbol{e}_{z} .
$$

$H_{K}$ is the anisotropy field, along the $x$-axis, $A$ is the exchange constant and the self-demagnetization field $K_{\perp} M_{z}$ is included.

In a stationary situation $\partial_{t} s=0$ from Eq. (2) one can obtain that

$$
\boldsymbol{N}=\frac{J_{e x}}{d_{F}} \boldsymbol{M} \times \boldsymbol{s}=\gamma \frac{d_{S}}{d_{F}} \partial_{j} \boldsymbol{J}_{j} .
$$

The spin current $\boldsymbol{J}_{j}$ in the superconductor is calculated in the framework of the Keldysh technique for quasiclassical Green's functions. All the technical details of the Green's function calculation are given in the Supplementary Material.

To understand the efficiency of the torque $\boldsymbol{N}$ induced by the presence of the superconductor, we compare its value to the characteristic value of the torque induced by the effective field $H_{\text {eff. }}$ Eq. (5) can be rewritten as $\boldsymbol{N} / \gamma H_{K} M=\zeta \partial_{\tilde{x}} \tilde{\boldsymbol{J}}_{x}$, with the dimensionless quantities $\partial_{\tilde{x}} \tilde{\boldsymbol{J}}_{x}=\left(2 e^{2} R_{N} v_{F} / \Delta_{0}^{2}\right) \partial_{x} \boldsymbol{J}_{x}$ and $\zeta=E_{S} / \pi E_{A}$. The latter is proportional to the ratio of the condensation energy $E_{S}=N_{F} \Delta_{0}^{2} d_{S} / 2$ and the anisotropy energy $E_{A}=M H_{K} d_{F} / 2$ per unit area of the film in the $(x, y)$-plane. Here and below $R_{N}=\pi /\left(2 e^{2} N_{F} v_{F}\right)$ is the normal state resistance of the film and $\Delta_{0}$ is the superconducting order parameter of the S film in the absence of the ferromagnet at zero temperature. Taking 


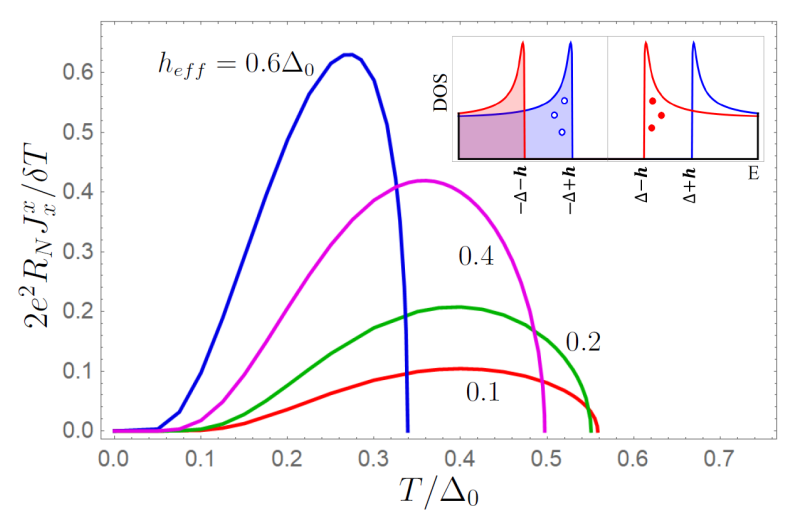

FIG. 2. Spin current divided by the temperature difference $\delta T \rightarrow 0$ in the homogeneous $\mathrm{S} / \mathrm{F}$ bilayer vs the temperature. $h_{\text {eff }}=0.1$ (red), 0.2(green), 0.4(purple), 0.6(blue) in units of $\Delta_{0}$. Insert: spin resolved DOS filled by thermally activated right-moving quasiparticles coming from the hot end. The spin-up $S_{x}=+1$ (spin down $S_{x}=-1$ ) DOS is blue (red). It is seen that all the right-moving quasiparticles contribute to spin flow of the same direction.

$E_{S} \sim d_{S} \times\left(10 \div 10^{3}\right) \mathrm{erg} / \mathrm{cm}^{3}$ (for conventional superconductors like $\mathrm{Al}$ and $\mathrm{Nb})$ and $E_{A} \sim d_{F} \times 10^{5} \mathrm{erg} / \mathrm{cm}^{3}$ for Py thin films 62, 63, or $E_{A} \sim d_{F} \times\left(10 \div 10^{2}\right) \mathrm{erg} / \mathrm{cm}^{3}$ for YIG thin films 64, we obtain that $\zeta$ can vary in a wide range $\zeta \sim\left(10^{-4} \div 10^{2}\right)\left(d_{S} / d_{F}\right)$.

Thermally induced spin current in a homogeneous $S / F$ bilayer. Now we are ready to calculate the thermally induced spin torque in the $\mathrm{S} / \mathrm{F}$ bilayer. But at first we discuss briefly thermally induced spin current in a $\mathrm{S} / \mathrm{F}$ bilayer with a homogeneous exchange field without a DW because it is a main ingredient of the torque providing the spin pumping into the DW region. Let us apply a temperature difference $T_{l}-T_{r}$ to the ends of the film. In this case a thermally induced spin current appears in the superconductor. This is a kind of a spin Seebeck effect. It is worth noting that the effect does not require an external spin source in the system as opposed to spin pumping experiments in superconductors [65, 66]. The spin current in the homogenous S/F bilayer only carries $x$-spin component $J_{x}^{x} \equiv J$, which is directed along the ferromagnet magnetization. Fig. 2 demonstrates the dependence of the spin current on the system temperature at small $\delta T=T_{l}-T_{r} \ll T$ for different $h$. For a homogeneous bilayer the spin thermopower at $\delta T / \Delta_{0} \ll 1$ is

$$
\frac{2 e^{2} R_{N} J}{\delta T}=F\left(\frac{\Delta+h}{2 T}\right)-F\left(\frac{\Delta-h}{2 T}\right)
$$

with $F(x)=x \tanh x-\ln \cosh x$. The maximal values of $2 e J R_{N} / \delta T$ are of the order of $\left(h / \Delta_{0}\right) \times 10^{-1} \mathrm{mV} / \mathrm{K}$ and are reached for $T \sim 0.6-0.7 T_{c}$, as illustrated in Fig. 2,

The estimated values of $2 e J R_{N} / \delta T$ are much larger than that ones obtained for nonsuperconducting systems containing metallic ferromagnets. Such large values of the spin Seebeck effect are a result of the huge spindependent electron-hole asymmetry close to the Fermi level, see the inset of Fig. 2, For the ballistic transport that we consider the distribution function of right-moving (left-moving) quasiparticles is determined by the Fermi distribution function of the left (right) end of the sample, which are assumed to be in thermal equilibrium at $T=T_{l(r)}$. Let us assume for simplicity that $T_{r}=0$. Then there are no left-moving quasiparticles. The spin-split DOS occupied by right-moving quasiparticles is shown in the insert to Fig. 2. We observe that at intermediate temperatures $\Delta-h<T<\Delta+h$ the spin-down DOS is presumably occupied by electron-like quasiparticles, while the spin-up DOS is occupied by hole-like quasiparticles. In this ideal situation all the thermally induced quasiparticles (both electrons and holes) have the same spin and contribute to the flow of spin-down quasiparticles to the right, which results in the maximal possible value of the thermally-induced spin current.

Thermally induced spin-transfer torque. The spatial profiles of the spin current in the presence of a plane DW (located in the $(x, y)$-plane) are presented in Fig. 3 for different temperatures of the hot end. At first, let us focus on $J_{x}^{x}$ component, which is the only nonzero component of the thermally induced spin current in the bulk. Due to the presence of two magnetic domains with opposite magnetizations it leads to spin pumping into the region occupied by the DW. This process is schematically illustrated on the top surface of Fig. 1 and is described there. At nonzero $T_{l}-T_{r}$ in-plane component $J_{x}^{y}$ also appears in the region of the DW. In the limit $T_{l}-T_{r} \rightarrow 0$ only $J_{x}^{z}$ survives. Then it represents a spontaneous spin current occuring in the region occupied by the wall in equilibrium. It is carried by the equal-spin Cooper pairs generated by the magnetic texture. Similar spontaneous spin currents have already been obtained, usually in a Josephson junction-type geometry 67-75]. The torque generated by this equilibrium spin current is compensated by the DW shape distortion resulting in additional contributions to the in-plane effective field (see supplemental material). Consequently, the equilibrium torque contribution does not effect the DW motion and is subtracted from nonequilibrium torque driving the wall.

The torque can be obtained via the spin current according to Eq. (5). In general, any spin torque can be written as $\boldsymbol{N}=a \partial_{x} \boldsymbol{m}+b \boldsymbol{m} \times \partial_{x} \boldsymbol{m}$, where $\boldsymbol{m}=\boldsymbol{M} / M$. The first (second) term can be related to electron spins following (being misaligned to) the magnetic texture. In the framework of the linear response theory the coefficients $a$ and $b$ are proportional to the temperature gradient. For the plane DW under consideration $N_{x}$ and $N_{y}$ components contribute to the adiabatic torque and $N_{z}$ gives rise to the nonadiabatic contribution.

The temperature dependence of the both adiabatic and nonadiabatic torques is determined by the spin pump- 

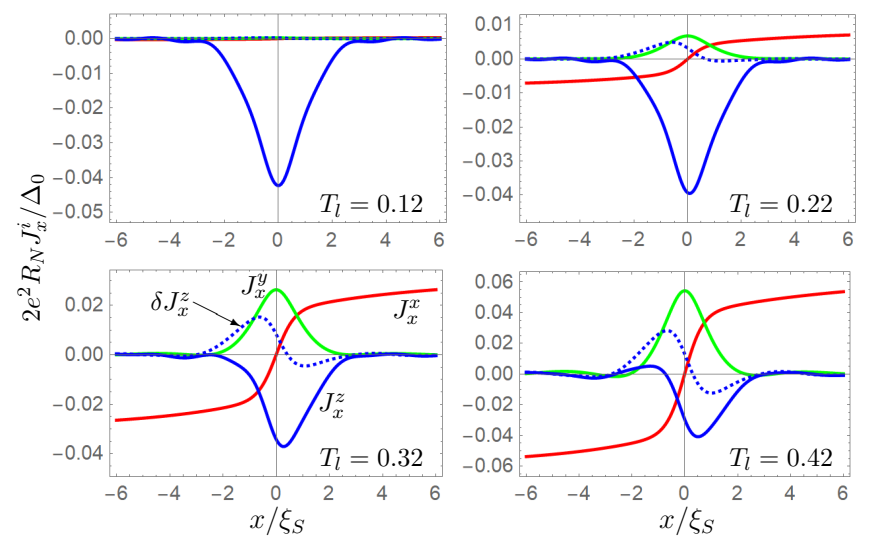

FIG. 3. Spatial profile of the spin current components $J_{x}^{x}$ (red), $J_{x}^{y}$ (green) and $J_{x}^{z}$ (blue) for different temperatures of the hot end, $\delta J_{x}^{z}=J_{x}^{z}-J_{x}^{z}\left(T_{l}=T_{r}\right)$. $h_{\text {eff }}=0.3 \Delta_{0}$, $T_{r}=0.02 \Delta_{0}, l_{D W}=0.5 \xi_{S}$, where $\xi_{S}=v_{F} / \Delta_{0}$ throughout the paper.

ing processes. It closely follows the temperature dependence of the bulk quasiparticle spin current (see supplemental material). Therefore, the spin pumping is the driving force of both adiabatic and nonadiabatic torque components. However, it is important to note that in the $\mathrm{S} / \mathrm{F}$ hybrid the nonadiabatic torque naturally appears because of two different length scales: $l_{D W}$ and $\xi_{S}$. A quasiparticle spin is aligned with the magnetization at the length scale $\sim \xi_{S}$. At $l_{D W} \lesssim \xi_{S}$ it inevitably mistracks magnetization giving rise to the nonadiabatic torque. The reason is that in the superconductor the quasiparticle is a coherent mixture of electron-like and hole-like excitations and strongly interacts with the condensate. Consequently, any changes of the quasiparticle spin are coupled to the changes in the equal-spin condensate wave function, which have characteristic spatial scale $\xi_{S}$. This is in contrast to the nonsuperconducting case, where the nonadiabatic torque is believed to be due to spin-flip scattering processes.

Thermally induced DW motion. The dynamics of the DW under the applied temperature difference is calculated from the LLG Eq. (3). At the present study we focus on small values of parameter $\zeta$ describing how strong is the torque induced by the superconductor. In this case we calculate the torque for the unperturbed DW neglecting the distortion of the DW shape due to its motion. Our numerical results for the spatial profiles of the moving DW are presented in the supplementary material and demonstrate that the distortion is indeed very small, therefore justifying the above assumption.

We found that for the values of $\zeta$ and $T_{l}-T_{r}$ considered in Fig. 4 the DW moves as a rigid object reaching the steady state at a characteristic time $t_{d}=1 / 4 \pi \alpha \gamma M$, that is the Walker's breakdown [76] is not reached in our calculation. For the considered parameters we have found

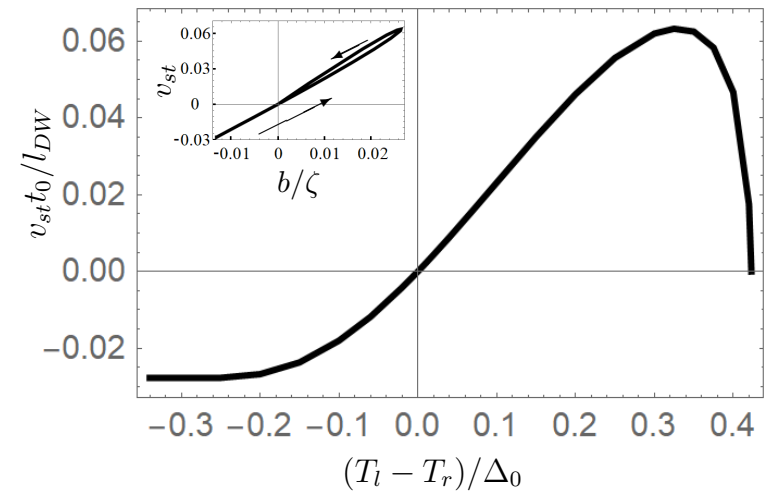

FIG. 4. DW velocity $v_{s t}$ as a function of $\delta T=T_{l}-T_{r}$. $t_{0}=\left(\gamma H_{K}\right)^{-1}$. Insert: $v_{s t}$ as a function of $b\left(x=x_{D W}\right)$. The direction of $\delta T$ growing along this curve is marked by the arrow. $\zeta=0.3, \alpha=0.2, K_{\perp}=H_{K} / M, T_{r}=0.35 \Delta_{0}$.

no sign of a precessional motion, which is typical for the motion in the regime after the Walker's breakdown. The DW velocity is calculated as $v(t)=\dot{x}_{D W}(t)$, where $x_{D W}(t)$ is the coordinate of the DW center at a given time and is extracted from the dynamical profiles of the magnetization. The steady state velocity $v_{s t}=v(t \rightarrow \infty)$ as a function of $\delta T$ is plotted in Fig. 4. We see that at $\delta T \ll \Delta_{0}$ the velocity is a linear function of the temperature difference. Although in superconducting systems the microscopically calculated coefficients $a$ and $b$ are spatially dependent (see supplementary material for details $), v_{s t} \sim b\left(x=x_{D W}\right)=-l_{D W} N_{z}\left(x=x_{D W}\right)$, as it is demonstrated in the insert to Fig. 4. It indicates that in this regime the DW motion is determined by the nonadiabatic torque analogously to the case of nonsuperconducting systems [77]. The "hysteretic behavior" of the parametric plot $v_{s t}(b)$ is due to the nonmonotonic dependence of the velocity, as well as $b\left(x_{D W}\right)$ on $\delta T$, which in turn results from the suppression of superconductivity by heating of the film.

The DW velocity $v_{s t}$ is linearly proportional to the $\mathrm{S} / \mathrm{F}$ coupling strength $\zeta$. At $\zeta=0.3$ and taking material parameters for Py films 63. $H_{K} \sim 500 \mathrm{Oe}$ and $l_{D W} \sim 20 \mathrm{~nm}$ or for YIG thin films $64 H_{K} \sim 0.5$ Oe and $l_{D W} \sim 1 \mu \mathrm{m}$ the maximal DW velocities can be estimated from Fig. 4 as $v_{P y} \sim 0.06\left(\alpha / \alpha_{P y}\right)\left(l_{D W} / t_{0}\right)_{P y}$, what gives us $v_{P y} \sim 100 \mathrm{~m} / \mathrm{s}$. Analogously, $v_{Y I G} \sim 10^{3} \mathrm{~m} / \mathrm{s}$. In these estimates we take into account that $v_{s t} \sim \alpha^{-1}$ and realistic values of $\alpha_{P y} \sim 0.01$ and $\alpha_{Y I G} \sim 10^{-4}$.

In summary, we have predicted and microscopically calculated a thermally induced STT in thin film S/F bilayers containing a DW. It features adiabatic as well as nonadiabatic contributions. The physical mechanism of the torque is a unique feature of superconducting hybrids: it results from (i) the extremely efficient quasiparticle spin pumping into the superconducting region close to the DW provided by the giant Seebeck effect and (ii) 
strong interaction between quasiparticles and the condensate in the superconductor resulting in the characteristic length scale $\xi_{S}$ of the quasiparticle spin evolution, what, in its turn, gives rise to a nonadiabatic torque contribution at $l_{D W} \lesssim \xi_{S}$. We have demonstrated that this torque allows for a high-velocity steady DW motion corresponding to $v \sim 100 \mathrm{~m} / \mathrm{s}$ at small temperature differences $\sim 1 K$ applied at the length of several domain wall widths.

This work was financially supported by the Deutsche Forschungsgemeinschaft (DFG) through SFB 767 Controlled Nanosystems. The work of A.M.B and I.V.B is carried out within the state task of ISSP RAS. I.V.B. also acknowledges the financial support by Foundation for the Advancement of Theoretical Physics and Mathematics "BASIS".

\section{SUPPLEMENTAL MATERIAL}

\section{Quasiclassical Keldysh Green's functions technique in terms of Riccati parametrization}

The matrix Green's function $\check{g}\left(\boldsymbol{r}, \boldsymbol{p}_{F}, \varepsilon, t\right)$ is a $8 \times 8$ matrix in the direct product of spin, particle-hole and Keldysh spaces and depends on the spatial vector $\boldsymbol{r}$, quasiparticle momentum direction $\boldsymbol{p}_{F}$, quasiparticle energy $\varepsilon$ and time $t$. In the S film it obeys the Eilenberger equation:

$$
i \boldsymbol{v}_{F} \nabla \check{g}\left(\boldsymbol{r}, \boldsymbol{p}_{F}\right)+\left[\varepsilon \tau_{z}+\boldsymbol{h}(\boldsymbol{r}) \boldsymbol{\sigma} \tau_{z}-\check{\Delta}, \check{g}\right]_{\otimes}=0,
$$

where $[A, B]_{\otimes}=A \otimes B-B \otimes A$ and $A \otimes B=\exp \left[(i / 2)\left(\partial_{\varepsilon_{1}} \partial_{t_{2}}-\right.\right.$ $\left.\left.\partial_{\varepsilon_{2}} \partial_{t_{1}}\right)\right]\left.A\left(\varepsilon_{1}, t_{1}\right) B\left(\varepsilon_{2}, t_{2}\right)\right|_{\varepsilon_{1}=\varepsilon_{2}=\varepsilon ; t_{1}=t_{2}=t} \cdot \tau_{x, y, z}$ are Pauli matrices in particle-hole space with $\tau_{ \pm}=\left(\tau_{x} \pm i \tau_{y}\right) / 2$. $\hat{\Delta}=\Delta(x) \tau_{+}-\Delta^{*}(x) \tau_{-}$is the matrix structure of the superconducting order parameter $\Delta(x)$ in the particle-hole space.

In the ballistic case, it is convenient to use the socalled Riccati parametrization for the Green's function [47, 48. In terms of the Riccati parametrization the retarded Green's function takes the form:

$$
\begin{aligned}
& \check{g}^{R, A}= \pm N^{R, A} \otimes \\
& \left(\begin{array}{cc}
1-\hat{\gamma}^{R, A} \otimes \hat{\tilde{\gamma}}^{R, A} & 2 \hat{\gamma}^{R, A} \\
2 \hat{\tilde{\gamma}}^{R, A} & -\left(1-\tilde{\tilde{\gamma}}^{R, A} \otimes \hat{\gamma}^{R, A}\right)
\end{array}\right), \\
& \check{g}^{K}=2 N^{R} \otimes \\
& \left(\begin{array}{cc}
x^{K}+\hat{\gamma}^{R} \otimes \hat{\tilde{x}}^{K} \otimes \hat{\tilde{\gamma}}^{A} & -\left(\hat{\gamma}^{R} \otimes \hat{\tilde{x}}^{K}-\hat{x}^{K} \hat{\gamma}^{A}\right) \\
\hat{\tilde{\gamma}}^{R} \otimes \hat{x}^{K}-\hat{\tilde{x}}^{K} \otimes \hat{\tilde{\gamma}}^{A} & \left.\hat{\tilde{x}}^{K}+\hat{\tilde{\gamma}}^{R} \otimes \hat{x}^{K} \otimes \hat{\gamma}^{A}\right)
\end{array}\right) \otimes N^{A}
\end{aligned}
$$

with

$$
N^{R, A}=\left(\begin{array}{cc}
1+\hat{\gamma}^{R, A} \otimes \hat{\tilde{\gamma}}^{R, A} & 0 \\
0 & 1+\hat{\tilde{\gamma}}^{R, A} \otimes \hat{\gamma}^{R, A}
\end{array}\right)^{-1}
$$

where $\hat{\gamma}^{R, A}, \hat{\tilde{\gamma}}^{R, A}, \hat{x}^{K}$ and $\hat{\tilde{x}}^{K}$ are matrices in spin space. Note that our parametrization differs from the definition in the literature [47, 48] by factors $i \sigma_{y}$ as $\hat{\gamma}_{\text {standard }}^{R, A}=\hat{\gamma}^{R, A} i \sigma_{y}$ and $\hat{\tilde{\gamma}}_{\text {standard }}^{R, A}=i \sigma_{y} \hat{\tilde{\gamma}}^{R, A}$. The Riccati parametrization Eqs. (8)-(10) obeys the normalization condition $\check{g} \otimes \check{g}=1$ automatically.

The Riccati amplitude $\hat{\gamma}$ obeys the following Riccatitype equations:

$i \boldsymbol{v}_{F} \nabla \hat{\gamma}^{R}+2 \varepsilon \hat{\gamma}^{R}=-\hat{\gamma}^{R} \otimes \Delta^{*} \otimes \hat{\gamma}^{R}-\left\{\boldsymbol{h} \boldsymbol{\sigma}, \hat{\gamma}^{R}\right\}_{\otimes}-\Delta$

and $\hat{\tilde{\gamma}}$ obeys the same equation with the substitution $\varepsilon \rightarrow$ $-\varepsilon, \boldsymbol{h} \rightarrow-\boldsymbol{h}$ and $\Delta \rightarrow \Delta^{*}$.

The distribution function $\hat{x}^{K}$ obeys the equation:

$$
\begin{array}{r}
i \boldsymbol{v}_{F} \nabla \hat{x}^{K}+i \partial_{t} \hat{x}^{K}+\hat{\gamma}^{R} \otimes \Delta^{*} \otimes \hat{x}^{K}+ \\
\hat{x}^{K} \otimes \Delta \otimes \hat{\tilde{\gamma}}^{A}+\left[\boldsymbol{h} \boldsymbol{\sigma}, \hat{x}^{K}\right]_{\otimes}=0,
\end{array}
$$

while $\hat{\tilde{x}}^{K}$ obeys the same equation with the substitution $\boldsymbol{h} \rightarrow-\boldsymbol{h}, \Delta \rightarrow \Delta^{*}, \hat{\gamma}^{R, A} \leftrightarrow \hat{\tilde{\gamma}}^{R, A}$. In this work, we assume $\Delta=\Delta^{*}$.

If we consider a locally spatially inhomogeneous magnetic texture like a domain wall, the Riccati amplitudes $\hat{\gamma}$ and $\hat{\tilde{\gamma}}$ can be found from Eq. 111 numerically with the following asymptotic condition:

$$
\begin{aligned}
\hat{\gamma}_{\infty}= & \gamma_{0 \infty}+\frac{\boldsymbol{h}_{\infty} \boldsymbol{\sigma}}{h} \gamma_{\infty}, \\
\gamma_{0 \infty}= & -\frac{1}{2}\left[\frac{\Delta}{\varepsilon+h+i \sqrt{\Delta^{2}-(\varepsilon+h)^{2}}}\right. \\
& \left.+\frac{\Delta}{\varepsilon-h+i \sqrt{\Delta^{2}-(\varepsilon-h)^{2}}}\right] \\
\gamma_{\infty}= & -\frac{1}{2}\left[\frac{\Delta}{\varepsilon+h+i \sqrt{\Delta^{2}-(\varepsilon+h)^{2}}}\right. \\
& \left.-\frac{\Delta}{\varepsilon-h+i \sqrt{\Delta^{2}-(\varepsilon-h)^{2}}}\right]
\end{aligned}
$$

and $\hat{\tilde{\gamma}}_{\infty}=-\hat{\gamma}_{\infty}$.

Eq. (11) is numerically stable if it is solved starting from $x=-\infty$ for right-going trajectories $v_{x}>0$ and from $x=+\infty$ for left-going trajectories $v_{x}<0$. On the contrary, $\hat{\tilde{\gamma}}$ can be found numerically starting from $x=+\infty$ for right-going trajectories $v_{x}>0$ and from $x=-\infty$ for left-going trajectories $v_{x}<0$. The advanced Riccati amplitudes can be found taking into account the relation [48] $\hat{\gamma}^{A}=-\left(\hat{\tilde{\gamma}}^{R}\right)^{\dagger}$. The superconducting order parameter is to be found self-consistently according to

$$
\Delta=-\frac{\lambda}{8} \int_{-\Omega}^{\Omega} d \varepsilon \operatorname{Tr}_{4}\left\langle\tau_{-} \check{g}^{K}\right\rangle
$$

where $\langle\ldots\rangle$ means averaging over the Fermi surface, and $\mathrm{Tr}_{4}$ is the trace in Nambu $\otimes$ Spin space. $\lambda$ is the coupling constant and $\Omega$ is the Debye frequency cutoff.

If we neglect the dependence of $\boldsymbol{h}$ on time, then it follows from Eq. 12 that the distribution function $\hat{x}^{K}$ for 
a given ballistic trajectory is determined by the equilibrium distribution function of the left (right) reservoir for $v_{F, x}>0\left(v_{F, x}<0\right)$ and takes the form

$$
\hat{x}_{ \pm}^{K}=\left(1+\hat{\gamma}_{ \pm}^{R} \otimes \hat{\tilde{\gamma}}_{ \pm}^{A}\right) \tanh \frac{\varepsilon}{2 T_{l, r}},
$$

where the subscript $+(-)$ corresponds to the trajectories $v_{F, x}>0\left(v_{F, x}<0\right)$. On the contrary,

$$
\hat{\tilde{x}}_{ \pm}^{K}=-\left(1+\hat{\tilde{\gamma}}_{ \pm}^{R} \otimes \hat{\gamma}_{ \pm}^{A}\right) \tanh \frac{\varepsilon}{2 T_{r, l}} .
$$

The terms $\propto \dot{\boldsymbol{h}}$ in Eq. 12 can be neglected under the conditions $(h / \Delta) v_{s t} / l_{D W} \Delta \ll 1$ and $(h / \Delta)\left(1 / t_{d} \Delta\right) \ll 1$, where $t_{d}$ is the characteristic time of the induced magnetization dynamics, $v_{s t}$ is the characteristic DW velocity and $l_{D W}$ is the DW width. For realistic parameters $t_{d} \sim 10^{-9}-10^{-8} c, v_{s t} \sim 100 \mathrm{~m} / \mathrm{s}$ according to our estimates. Therefore, at $\Delta \sim 1 K$ and $h / \Delta \lesssim 1$ these conditions are fulfilled to a good accuracy for any experimentally reasonable DW width $l_{D W} \sim 10 \mathrm{~nm}-1 \mu \mathrm{m}$. Physically, these terms account for the electromotive force, which arises in the system due to the magnetization dynamics and has been studied in different contexts before [49 61, but here its back influence on the magnetization dynamics can be safely neglected.

The quantity of the main interest for us is the spin current flowing in the superconductor. It exerts a torque on the ferromagnet magnetization. The spin current of spin projection $\boldsymbol{J}$ in direction $j$ can be calculated as follows:

$$
\boldsymbol{J}_{j}=-\frac{N_{F}}{16} \int_{-\infty}^{\infty} d \varepsilon \operatorname{Tr}_{4}\left[\boldsymbol{\sigma}\left\langle v_{F, j} \check{g}^{K}\right\rangle\right],
$$

where $\check{g}^{K}\left(\epsilon, \boldsymbol{v}_{F}\right)$ represents the Keldysh part of the quasiclassical Green's function. $N_{F}$ is the normal density of states at the Fermi level.

\section{Spontaneous spin current, DW magnetization profile and self-consistent superconducting order parameter in equilibrium $\mathrm{S} / \mathrm{F}$ bilayer}

The spontaneous spin current for our ballistic S film in proximity to the ferromagnet with a coplanar DW is plotted in Fig. 5. It is seen that the amplitude of the spontaneous current is higher for narrow DWs. In the limit $l_{D W} / \xi_{S} \gg 1$ it disappears. The spin current is not conserved and exerts a spin-transfer torque on the magnetization. We find the resulting equilibrium shape of the DW accounting for the spin-transfer torque from the LLG equation. It is found that the presence of the superconductor results in the appearance of the additional oscillations of the magnetization in the $(x, y)$-plane. These oscillations generate additional contributions to the inplane effective field, which exerts a $z$-directed torque on the magnetization, thus compensating the action of the

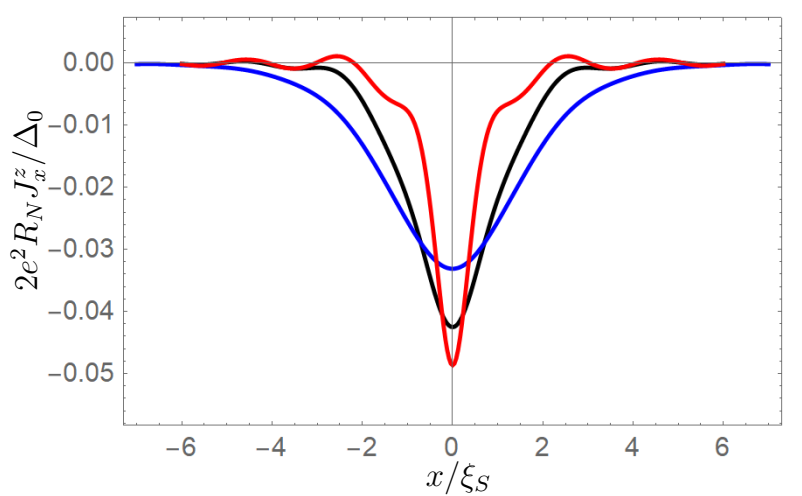

FIG. 5. Spin current $J_{x}^{z}(x)$ at the plane DW in equilibrium. Other components of the spin current are zero. $l_{D W}=\xi_{S}$ (blue); $0.5 \xi_{S}$ (black); $0.2 \xi_{S}$ (red). $h_{\text {eff }}=0.3 \Delta_{0}$, $T=0.02 \Delta_{0}$.

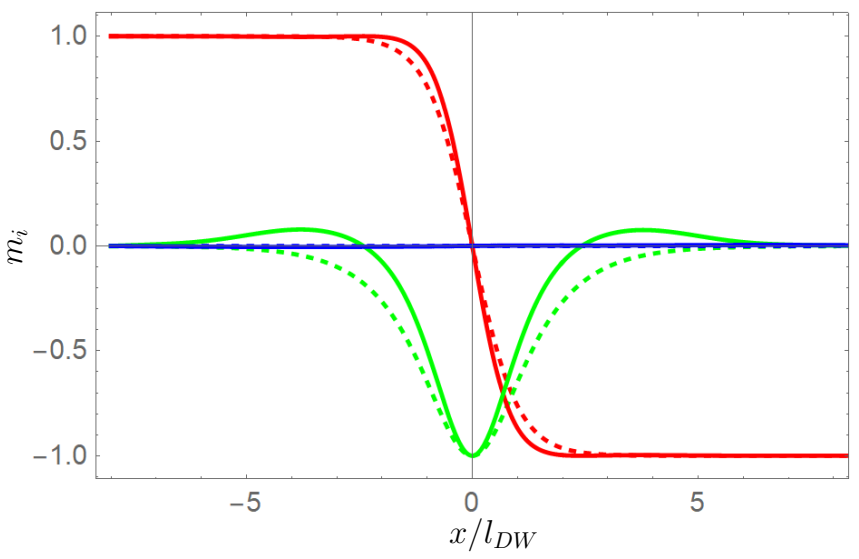

FIG. 6. Magnetization profile in the equilibrium S/F bilayer for $\zeta=10 . \boldsymbol{m}_{i}=\boldsymbol{M}_{i} / M$. Different magnetization components are plotted in different colors: $m_{x}$-red, $m_{y}$-green and $m_{z}$-blue. The dotted curves correspond to $\zeta=0$ (the superconductor is absent). $l_{D W}=0.5 \xi_{S}, h_{\text {eff }}=0.3 \Delta_{0}$, $T=0.02 \Delta_{0}, K_{\perp}=H_{K} / M$.

spontaneous spin current. The resulting magnetization profile in equilibrium is presented in Fig. 6. For realistic ratios of the anisotropy field to the demagnetization field $H_{K} / K_{\perp} M$ we have found no noticeable deviation of the DW shape from the initial $(x, y)$-plane. At the same time the distortion of the DW is accompanied by its narrowing, which also appears to provide appropriate contributions to the in-plane effective field. It is worth noting that Fig. 6 is plotted for the extremely high value of $\zeta=10$ to make the distortions clearly visible.

Equilibrium S/F bilayer with a DW was previously considered for a dirty system in Ref. 75 based on the free energy consideration. For a trial Neel-type plane DW it was found that the presence of the superconductor shrinks the DW size. This effect is closely connected to the fact that the the superconductivity can be enhanced 


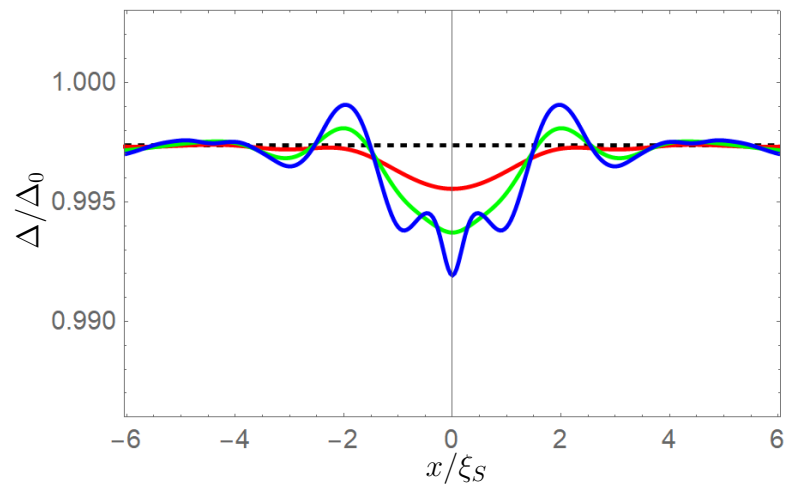

FIG. 7. Self-consistent order parameter as a function of the spatial coordinate along the bilayer. $l_{D W}=\xi_{S}$ (red); $0.5 \xi_{S}$ (green); $0.2 \xi_{S}$ (blue). $h_{e f f}=0.3 \Delta_{0}$. Black dotted line represents the order parameter value in the $\mathrm{S} / \mathrm{F}$ bilayer in the absence of a DW.

for narrow DWs due to the effective averaging of the exchange field, and, consequently, to weaker suppression of superconductivity in the DW region. The increase of the superconducting order parameter provides the corresponding gain in the condensation energy. Here we do not see this effect because the considered exchange fields and temperatures are too small to cause essential suppression of the order parameter far from the DW, what is a necessary condition to have the superconductivity enhancement (restoring) near the DW. Instead, we observe weak Friedel-like oscillations of the order parameter near the DW and suggest that it is a specific feature of the ballistic limit we consider.

The self-consistent profile of the order parameter calculated in the presence of a DW in the F layer is demonstrated in Fig. 7 for three different DW widths. It is seen that it manifests Friedel-like oscillating behavior. The oscillations become more pronounced for narrow DWs, but are generally weak for considered values of the suppression factors: exchange field and temperature.

\section{Details of the order parameter and torque calculations under the applied temperature difference}

At first in Fig. 8 we demonstrate results for the selfconsistent profile of the superconducting order parameter in the presence of the DW and a temperature difference. The small overall suppression of the order parameter by heating of the superconductor is clearly seen. More interesting feature is that the order parameter is additionally suppressed near the DW from the "hotter" side and slightly enhanced with respect to the bulk value from the "colder" side of the DW. It seems that there appears an excess (lack) of quasiparticles at the corresponding side of the DW.

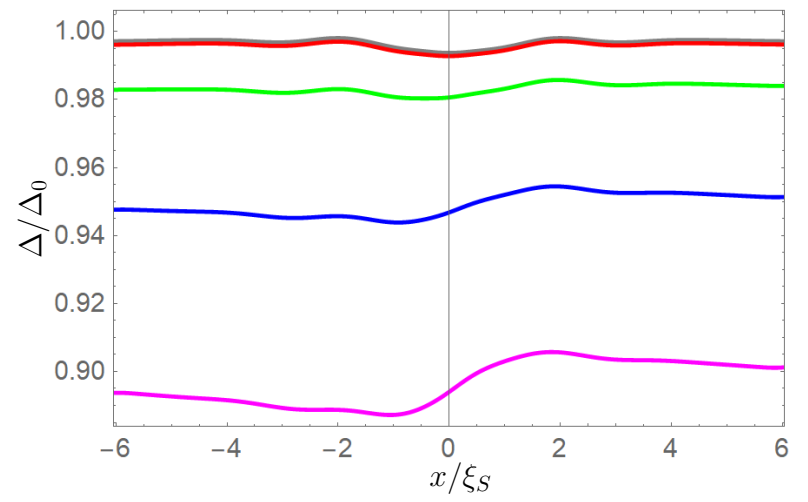

FIG. 8. Self-consistent profile of the order parameter in the presence of the DW and a temperature difference. Different curves correspond to different temperatures of the hot end $T_{l}=0.02 \Delta_{0}$ (grey), 0.12(red), 0.22(green), 0.32(blue) and 0.42 (purple). $h_{e f f}=0.3 \Delta_{0}, T_{r}=0.02 \Delta_{0}, l_{D W}=0.5 \xi_{S}$.
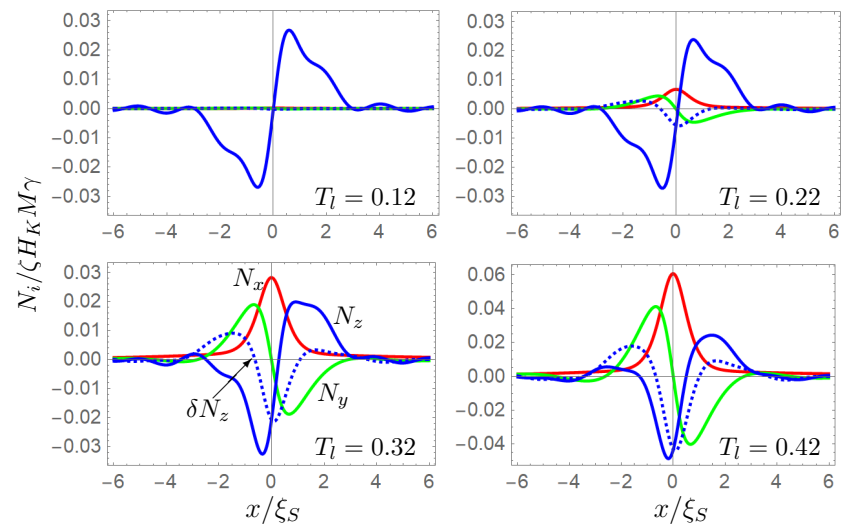

FIG. 9. Spatial profile of the torque components $N_{x}$ (red), $N_{y}$ (green) and $N_{z}$ (blue) at the plane DW under the applied heat bias, $\delta N_{z}=N_{z}-N_{z}\left(T_{l}=T_{r}\right) . h_{e f f}=0.3 \Delta_{0}, T_{r}=0.02 \Delta_{0}$, $l_{D W}=0.5 \xi_{S}$.

The spatial profiles of the torque $\boldsymbol{N}$ acting on the DW from the spatially-dependent spin current, shown in Fig. 3 of the main text, are presented in Fig. 9. The torque exhibit strong temperature dependence. In order to investigate this dependence in more detail, in Figs. 10(b)-(d) we present the torque components at different temperatures normalized to the value of the bulk thermally-induced spin current at a given temperature difference. It is seen that the temperature dependence of the torque closely follows the temperature dependence of the bulk thermally-induced spin current, what means that the torque is indeed driven by the thermal spin pumping process, described in the caption to Fig. 1 of the main text. Panels(b)-(d) of Fig. 10 represent all three torque components. The particular temperature differences are encoded by colors and marked by the circles in Fig. 10(a).

In order to investigate the spatial structure of the 

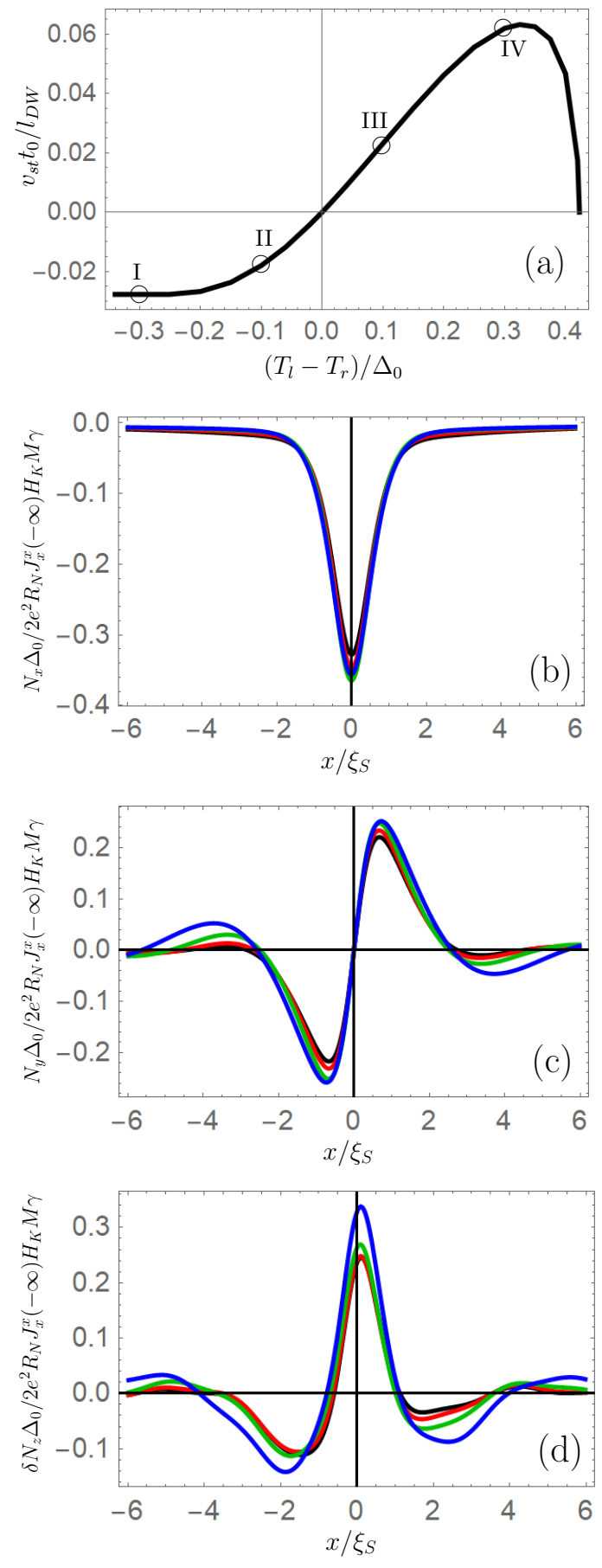

FIG. 10. (a) DW velocity as a function of the temperature difference. All the parameters are the same as in the main text. (b)-(d) Spatial profiles of the torque components, normalized to the bulk thermally-induced spin current. Different curves correspond to different temperature differences, marked by circles in panel (a): I-black; II-red; III-green; IV-blue.

torque induced by the presence of the superconductor, we separate it to adiabatic $a \partial_{x} \boldsymbol{m}$ and nonadiabatic $b \boldsymbol{m} \times \partial_{x} \boldsymbol{m}$ contributions. In fact, the full microscopic re-
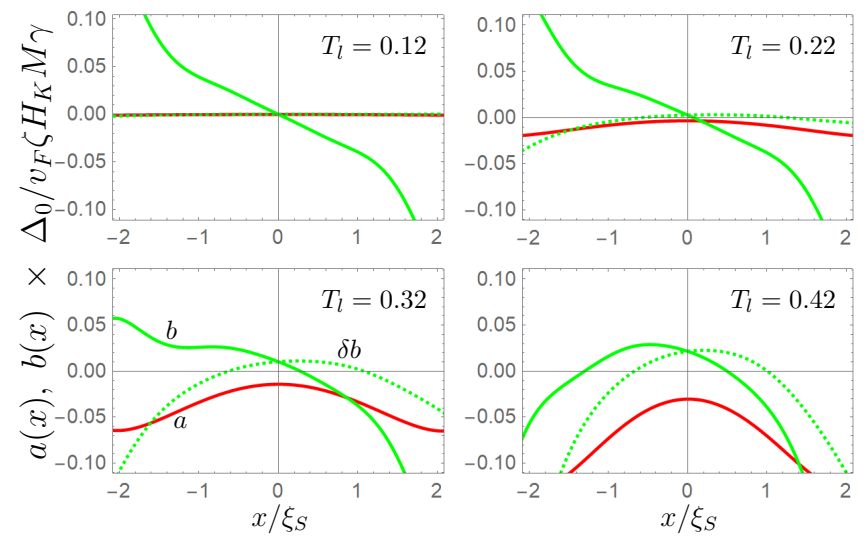

FIG. 11. Torque coefficients $a(x)$ (red) and $b(x)$ (green) for different temperatures of the hot end, $\delta b=b-b\left(T_{l}=T_{r}\right)$. $h_{\text {eff }}=0.3 \Delta_{0}, T_{r}=0.02 \Delta_{0}, l_{\mathrm{DW}}=0.5 \xi_{S}$.
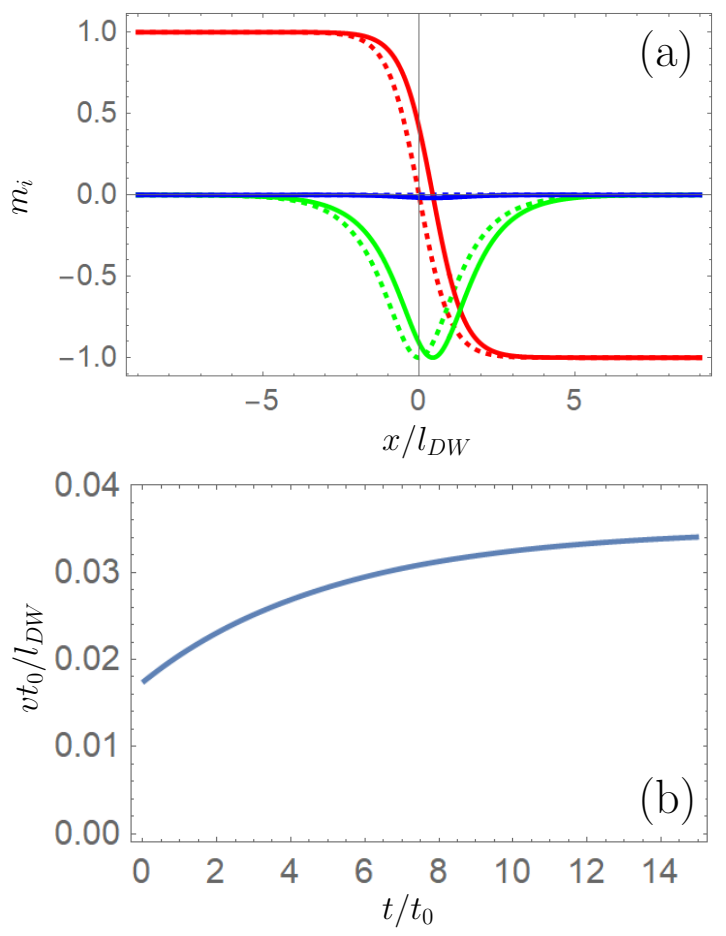

FIG. 12. (a) Components of the magnetization profile at $t=0$ (dashed lines) and at $t=15 t_{0}$ (solid lines). $m_{x}$-red; $m_{y^{-}}$ green and $m_{z}$-blue. (b) the DW velocity as a function of time. $\zeta=0.3, \alpha=0.2, K_{\perp}=H_{K} / M, T_{r}=0.35 \Delta_{0}$ and $T_{l}=0.50 \Delta_{0}$.

sult obtained from Eq. (5) of the main text also contains the term $\sim \boldsymbol{m}$, but we exclude this contribution because in the framework of the considered model the amplitude of the magnetization is fixed.

The essential feature of the superconducting system is that the microscopically calculated coefficients $a$ and $b$ in our case are spatially dependent. They are plotted in 
Fig. 11 as functions of $x$-coordinate (the DW center is at $x=0$ ) for different temperatures of the hot end. It is different from the nonsuperconducting case, where they are typically do not depend on coordinates due to absence of a corresponding spatial scale. Here the characteristic scale of the spatial variation of $a$ and $b$ is determined by the superconducting coherence length $\xi_{S}$. It is interesting that the coefficient $b$ is even a sign-changing function of the $x$-coordinate. In equilibrium, at $T_{l}=T_{r}, b=0$ at $x=x_{D W}$, that is, in the center of the DW. Under the applied temperature difference it is useful to introduce $\delta b=b-b\left(T_{l}=T_{r}\right)$. It becomes nonzero at $x=x_{D W}$ : $\delta b\left(x=x_{D W}\right)=b\left(x=x_{D W}\right) \neq 0$. The DW velocity is proportional to this quantity as it is demonstrated in the main text.

\section{Magnetization dynamics and details of the DW velocity calculations}

Fig. 12 represents all the three Cartesian components of the magnetization profile as functions of $x$-coordinate at the initial moment $t=0$ when the tempetature difference is applied and at some $t_{f}>0$ when the DW motion practically reaches stationary regime. After examining the dynamics between $t=0$ and $t_{f}$ we concluded that the DW moves as a rigid object keeping its initial shape to a good accuracy. Therefore, we are in the regime before the Walker's breakdown. Fig. 12(a) demonstrates that the distortions of the DW shape are indeed negligible.

In the regime below Walker's breakdown the DW center position $x_{D W}$ is a well-defined quantity and the DW velocity can be extracted from the numerically calculated magnetization profiles as $v=\dot{x}_{D W}$. The DW velocity $v$ as a function of time is presented in Fig. 12(b).

[1] G. Bauer, E. Saitoh, and B.J. van Wees, Spin caloritronics, Nat. Mat. 11, 391 (2012).

[2] L. Berger, Thermal forces on ferromagnetic domain walls, associated with the wall entropy, J. Appl. Phys. 58, 450 (1985).

[3] S. U. Jen and L. Berger, Thermal domain drag effect in amorphous ferromagnetic materials. I. Theory, J. Appl. Phys. 59, 1278 (1986).

[4] M. Hatami, G. E.W. Bauer, Q. Zhang and P. J. Kelly, Thermal Spin-Transfer Torque in Magnetoelectronic Devices, Phys. Rev. Lett. 99, 066603 (2007).

[5] A. A. Kovalev and Y. Tserkovnyak, Thermoelectric spin transfer in textured magnets, Phys. Rev. B 80, 100408 (2009).

[6] D. Hinzke and U. Nowak, Domain Wall Motion by the Magnonic Spin Seebeck Effect, Phys. Rev. Lett. 107, 027205 (2011).

[7] P. Yan, X. S. Wang, and X. R. Wang, All-Magnonic Spin-
Transfer Torque and Domain Wall Propagation, Phys. Rev. Lett. 107, 177207 (2011).

[8] K. M.D. Hals, A. Brataas, and G. E.W. Bauer, Thermopower and thermally induced domain wall motion in (Ga, Mn)As, Solid State Comm. 150, 461 (2010).

[9] S. Moretti, V. Raposo, E. Martinez, and L.s Lopez-Diaz, Domain wall motion by localized temperature gradients, Phys. Rev. B 95, 064419 (2017).

[10] S. U. Jen and L. Berger, Thermal domain drag effect in amorphous ferromagnetic materials. II. Experiments, J. Appl. Phys. 59, 1285 (1986).

[11] J. Torrejon, G. Malinowski, M. Pelloux, R. Weil, A. Thiaville, J. Curiale, D. Lacour, F. Montaigne, and M. Hehn, Unidirectional Thermal Effects in Current-Induced Domain Wall Motion, Phys. Rev. Lett. 109, 106601 (2012).

[12] W. Jiang, P. Upadhyaya, Y. Fan, J. Zhao, M. Wang, Li-Te Chang, M. Lang, K. L. Wong, M. Lewis, Y.-T. Lin, J. Tang, S. Cherepov, X. Zhou, Y. Tserkovnyak, R. N. Schwartz, and K. L. Wang, Direct Imaging of Thermally Driven Domain Wall Motion in Magnetic Insulators, Phys. Rev. Lett. 110, 177202 (2013).

[13] A. J. Ramsay, P. E. Roy, J. A. Haigh, R. M. Otxoa, A. C. Irvine, T. Janda, R. P. Campion, B. L. Gallagher, and J. Wunderlich, Optical Spin-Transfer-Torque-Driven Domain-Wall Motion in a Ferromagnetic Semiconductor, Phys. Rev. Lett. 114, 067202 (2015).

[14] H. Yu, S. Granville, D. P. Yu, and J.-Ph. Ansermet, Evidence for Thermal Spin-Transfer Torque, Phys. Rev. Lett. 104, 146601 (2010).

[15] A. Pushp, T. Phung, C. Rettner, B. P. Hughes, See-Hun Yang, and S. S. P. Parkin, Giant thermal spin-torqueassisted magnetic tunnel junction switching, PNAS 112, 6585 (2015).

[16] M. Johnson and R. H. Silsbee, Thermodynamic analysis of interfacial transport and of the thermomagnetoelectric system, Phys. Rev. B 35, 4959 (1987); M. Johnson, Charge-Spin Coupling at a Ferromagnet-Nonmagnet Interface, J. Supercond. 16, 679 (2003).

[17] L. Gravier, S. Serrano-Guisan, F. Reuse, and J.-P. Ansermet, Thermodynamic description of heat and spin transport in magnetic nanostructures, Phys. Rev. B 73, 024419 (2006); Spin-dependent Peltier effect of perpendicular currents in multilayered nanowires, Phys. Rev. B 73, 052410 (2006).

[18] K. Uchida, J. Xiao, H. Adachi, J. Ohe, S. Takahashi, J. Ieda, T. Ota, Y. Kajiwara, H. Umezawa, H. Kawai, G. E. W. Bauer, S. Maekawa, and E. Saitoh, Spin Seebeck insulator, Nature Mater., 9, 894 (2010).

[19] A. Slachter, F. Bakker, J. Adam, and B.J. van Wees, Thermally driven spin injection from a ferromagnet into a non-magnetic metal, Nature Phys. 6, 879 (2010).

[20] K. Uchida, S. Takahashi, K. Harii, W. Koshibae, K. Ando, S. Maekawa and E. Saitoh, Observation of the spin Seebeck effect, Nature, 455, 778 (2008).

[21] M. Hatami, G. E.W. Bauer, Q. Zhang and P. J. Kelly, Thermoelectric effects in magnetic nanostructures, Phys. Rev. B 79, 174426 (2009).

[22] P. Machon, M. Eschrig, and W. Belzig, Nonlocal Thermoelectric Effects and Nonlocal Onsager relations in a Three-Terminal Proximity-Coupled SuperconductorFerromagnet Device, Phys. Rev. Lett. 110, 047002 (2013).

[23] M. S. Kalenkov, A. D. Zaikin, and L. S. Kuzmin, Theory of a Large Thermoelectric Effect in Superconductors 
Doped with Magnetic Impurities, Phys. Rev. Lett. 109, 147004 (2012).

[24] A. Ozaeta, P. Virtanen, F.S. Bergeret, and T.T. Heikkila, Predicted Very Large Thermoelectric Effect in Ferromagnet-Superconductor Junctions in the Presence of a Spin-Splitting Magnetic Field, Phys. Rev. Lett. 112, 057001 (2014).

[25] F. Giazotto, J. W. A. Robinson, J. S. Moodera, and F. S. Bergeret, Proposal for a phase-coherent thermoelectric transistor, Appl. Phys. Lett. 105, 062602 (2014).

[26] M. S. Kalenkov and A. D. Zaikin, Enhancement of thermoelectric effect in diffusive superconducting bilayers with magnetic interfaces, Phys. Rev. B 91, 064504 (2015).

[27] P. Machon, M. Eschrig, and W.Belzig, Giant thermoelectric effects in a proximity-coupled superconductor-ferromagnet device, New J. Phys. 16, 073002 (2014).

[28] S. Kawabata, A. Ozaeta, A. S. Vasenko, F. W. J. Hekking, and F. S. Bergeret, Efficient electron refrigeration using superconductor/spin-filter devices, Appl. Phys. Lett. 103, 032602 (2013).

[29] F. Giazotto, T. T. Heikkila, and F. S. Bergeret, Very Large Thermophase in Ferromagnetic Josephson Junctions, Phys. Rev. Lett. 114, 067001 (2015).

[30] J. Linder, M.E. Bathen, Spin caloritronics with superconductors: Enhanced thermoelectric effects, generalized Onsager response-matrix, and thermal spin currents, Phys. Rev. B 93, 224509 (2016).

[31] I. V. Bobkova and A. M. Bobkov, Thermospin effects in superconducting heterostructures, Phys. Rev. B 96, 104515 (2017).

[32] A Rezaei, A. Kamra, P. Machon, and W. Belzig, Spin-flip enhanced thermoelectricity in superconductorferromagnet bilayers, New J. Phys. 20, 073034 (2018).

[33] F. Aikebaier, M. A. Silaev, T. T. Heikkila, Supercurrentinduced charge-spin conversion in spin-split superconductors, Phys. Rev. B 98, 024516 (2018).

[34] S. Kolenda, M. J. Wolf, and D. Beckmann, Observation of Thermoelectric Currents in High-Field Superconductor-Ferromagnet Tunnel Junctions, Phys. Rev. Lett. 116, 097001 (2016).

[35] S. Kolenda, C. Surgers, G. Fischer, and D. Beckmann, Thermoelectric effects in superconductor-ferromagnet tunnel junctions on europium sulfide, Phys. Rev. B 95 224505 (2017).

[36] S. Kolenda, P. Machon, D. Beckmann, and W. Belzig, Nonlinear thermoelectric effects in high-field superconductor-ferromagnet tunnel junctions, Beilstein J. Nanotechnol. 71579 (2016).

[37] Y. Pu, D. Chiba, F. Matsukura, H. Ohno, J Shi, Mott Relation for Anomalous Hall and Nernst Effects in $G a_{1-x} M n_{x} A s$ Ferromagnetic Semiconductors, Phys. Rev. Lett. 101117208 (2008).

[38] A. I. Buzdin, Proximity effects in superconductorferromagnet heterostructures, Rev. Mod. Phys. 77, 935 (2005).

[39] L. N. Bulaevskii, A. I. Buzdin, and S. V. Panjukov, The oscillation dependence of the critical current on the exchange field of ferromagnetic metals (F) in Josephson junction S-F-S, Solid State Commun. 44, 539 (1982).

[40] W. Belzig, A. Brataas, Y. Nazarov, and G. Bauer, Spin accumulation and Andreev reflection in a mesoscopic ferromagnetic wire, Phys. Rev. B 62, 9726 (2000).

[41] F.S. Bergeret, A.F. Volkov, and K.B. Efetov, Odd triplet superconductivity and related phenomena in superconductor-ferromagnet structures, Rev. Mod. Phys. 4, 1321 (2005).

[42] T. Tokuyasu, J. A. Sauls, and D. Rainer, Proximity effect of a ferromagnetic insulator in contact with a superconductor, Phys. Rev. B 38, 8823 (1988).

[43] A. Millis, D. Rainer, and J. A. Sauls, Quasiclassical theory of superconductivity near magnetically active interfaces, Phys. Rev. B 38, 4504 (1988).

[44] A. Cottet, D. Huertas-Hernando, W. Belzig, and Yu.V. Nazarov, Spin-dependent boundary conditions for isotropic superconducting Green's functions, Phys. Rev. B 80, 184511 (2009) [Erratum: Phys. Rev. B 83, 139901(E) (2011)].

[45] M. Eschrig, A. Cottet, W. Belzig, J. Linder, General boundary conditions for quasiclassical theory of superconductivity in the diffusive limit: application to strongly spin-polarized systems, New J. Phys. 17, 083037 (2015).

[46] A. Kamra, A. Rezaei, and W. Belzig, Spin Splitting Induced in a Superconductor by an Antiferromagnetic Insulator, Phys. Rev. Lett. 121, 247702 (2018).

[47] M. Eschrig, Distribution functions in nonequilibrium theory of superconductivity and Andreev spectroscopy in unconventional superconductors, Phys. Rev. B 61, 9061 (2000).

[48] M. Eschrig, Scattering problem in nonequilibrium quasiclassical theory of metals and superconductors: General boundary conditions and applications, Phys. Rev. B 80, 134511 (2009).

[49] A. Stern, Berry's phase, motive forces, and mesoscopic conductivity, Phys. Rev. Lett. 68, 1022 (1992).

[50] M. Stone, Magnus force on skyrmions in ferromagnets and quantum Hall systems, Phys. Rev. B 53, 16573 (1996).

[51] G. E. Volovik, Linear momentum in ferromagnets, J. Phys. C 20, L83 (1987).

[52] L. Berger, Possible existence of a Josephson effect in ferromagnets, Phys. Rev. B 33, 1572 (1986).

[53] S.E. Barnes and S. Maekawa, Generalization of Faraday's Law to Include Nonconservative Spin Forces, Phys. Rev. Lett. 98, 246601 (2007).

[54] R. A. Duine, Spin pumping by a field-driven domain wall, Phys. Rev. B 77, 014409 (2008).

[55] W. M. Saslow, Spin pumping of current in non-uniform conducting magnets, Phys. Rev. B 76, 184434 (2007).

[56] Y. Tserkovnyak and M. Mecklenburg, Electron transport driven by nonequilibrium magnetic textures, Phys. Rev. B 77, 134407 (2008).

[57] S. Zhang and S. S.-L. Zhang, Generalization of the Landau-Lifshitz-Gilbert Equation for Conducting Ferromagnets, Phys. Rev. Lett. 102, 086601 (2009).

[58] S. A. Yang, G. S. D. Beach, C. Knutson, Di Xiao, Q. Niu, M. Tsoi, and J. L. Erskine, Universal Electromotive Force Induced by Domain Wall Motion, Phys. Rev. Lett. 102, 067201 (2009).

[59] S. A. Yang, G. S. D. Beach, C. Knutson, Di Xiao, Z. Zhang, M. Tsoi, Q. Niu, A. H. MacDonald, and J. L. Erskine, Topological electromotive force from domainwall dynamics in a ferromagnet, Phys. Rev. B 82, 054410 (2007).

[60] D.S. Rabinovich, I.V. Bobkova, A.M. Bobkov, and M.A. Silaev, Resistive state of SFS Josephson junctions in the presence of moving domain walls, Phys. Rev. Lett. 123, 207001 (2019) 
[61] D.S. Rabinovich, I.V. Bobkova, A.M. Bobkov, Electrical response of S-F-TI-S junctions on magnetic texture dynamics, Phys. Rev. B 101, 054517 (2020)

[62] G. S. D. Beach, C. Nistor, C. Knutson, M. Tsoi and J. L. Erskine, Dynamics of field-driven domain-wall propagation in ferromagnetic nanowires, Nat. Mater. 4, 741 (2005).

[63] G. S. D. Beach, C. Knutson, C. Nistor, M. Tsoi, and J. L. Erskine, Nonlinear Domain-Wall Velocity Enhancement by Spin-Polarized Electric Current, Phys. Rev. Lett. 97, 057203 (2006).

[64] J. Mendil, M. Trassin, Q. Bu, J. Schaab, M. Baumgartner, C. Murer, P. T.Dao, J. Vijayakumar, D. Bracher, C. Bouillet, C. A. F. Vaz, M. Fiebig, and P. Gambardella, Magnetic properties and domain structure of ultrathin yttrium iron garnet/Pt bilayers, Phys. Rev. Mat. 3, 034403 (2019).

[65] Y. Yao, Qi Song, Y. Takamura, J. P. Cascales, W. Yuan, Y. Ma, Yu Yun, X. C. Xie, J. S. Moodera, and Wei Han, Probe of spin dynamics in superconducting $\mathrm{NbN}$ thin films via spin pumping, Phys. Rev. B 97, 224414 (2018).

[66] M. Umeda, Y. Shiomi, T. Kikkawa, T. Niizeki, J. Lustikova, S. Takahashi, and E. Saitoh, Spin-current coherence peak in superconductor/magnet junctions, Appl. Phys. Lett. 112, 232601 (2018).

[67] I. V. Bobkova, Yu. S. Barash, Effects of spin-orbit interaction on superconductor - ferromagnet heterostructures: spontaneous electric and spin surface currents, Pisma v Zh. Eksper. Teoret. Fiz. 80, 563 (2004); JETP Lett. 80, 494 (2004).
[68] R. Grein, M. Eschrig, G. Metalidis, and Gerd Schon, Spin-Dependent Cooper Pair Phase and Pure Spin Supercurrents in Strongly Polarized Ferromagnets, Phys. Rev. Lett. 102, 227005 (2009).

[69] M. Alidoust, J. Linder, G. Rashedi, T. Yokoyama, and A. Sudbo, Spin-polarized Josephson current in superconductor/ferromagnet/superconductor junctions with inhomogeneous magnetization, Phys. Rev. B 81, 014512 (2010).

[70] Z. Shomali, M. Zareyan, W. Belzig, Spin supercurrent in Josephson contacts with noncollinear ferromagnets, New J. Phys. 13, 083033 (2011).

[71] M. Alidoust, and K. Halterman, Spontaneous edge accumulation of spin currents in finite-size two-dimensional diffusive spin-orbit coupled SFS heterostructures, New J. Phys. 17, 033001 (2015).

[72] K. Halterman, O.T. Valls, and C.-T. Wu, Charge and spin currents in ferromagnetic Josephson junctions, Phys. Rev. B 92, 174516 (2015).

[73] S. Jacobsen, I. Kulagina, J. Linder, Controlling superconducting spin flow with spin-flip immunity using a single homogeneous ferromagnet, Sci. Rep. 6, 23926 (2016).

[74] F. Konschelle, I. V. Tokatly, F. S. Bergeret, Ballistic Josephson junctions in the presence of generic spin dependent fields, Phys. Rev. B 94, 014515 (2016).

[75] F. Aikebaier, P. Virtanen, and T. Heikkila, Superconductivity near a magnetic domain wall, Phys. Rev. B 99, 104504 (2019).

[76] N. L. Schryer and L. R. Walker, The motion of $180^{\circ}$ domain walls in uniform dc magnetic fields, J. Appl. Phys., 455406 (1974).

[77] Z. Li, J. He, and S. Zhang, Effects of spin current on ferromagnets, J. Appl. Phys. 99, 08Q702 (2006). 